UStథU孔3U乙 <UUUh4

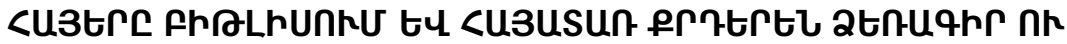

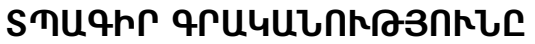

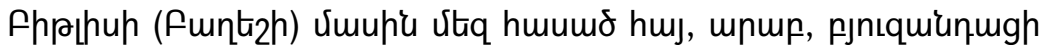

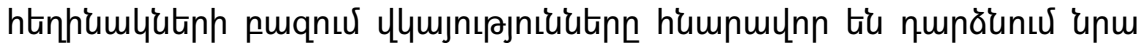

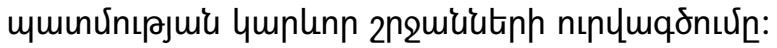

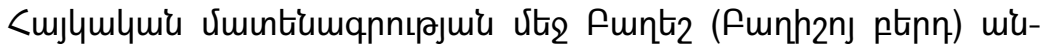

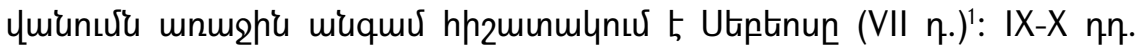

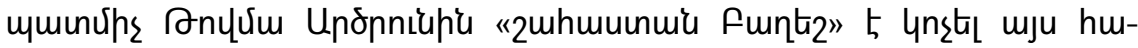
pniuun 2nquiun':

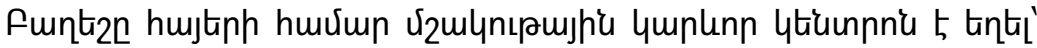

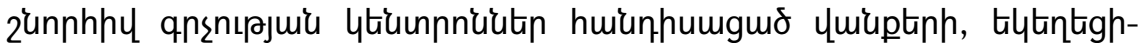

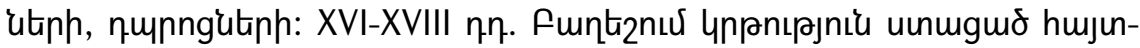

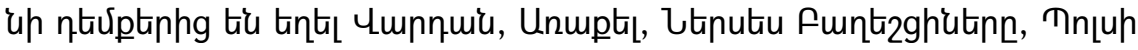

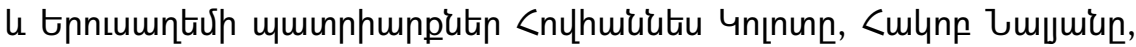

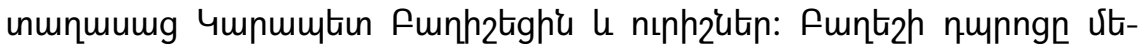

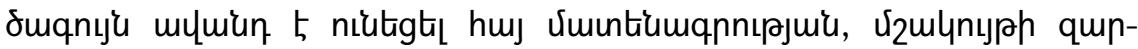

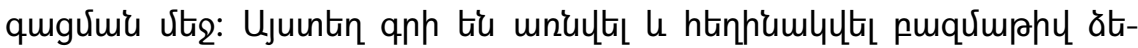

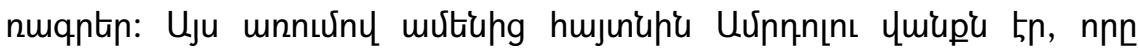

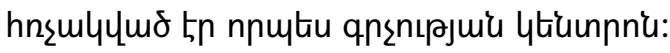

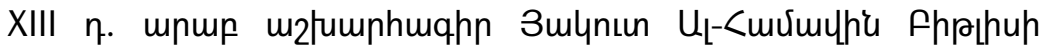

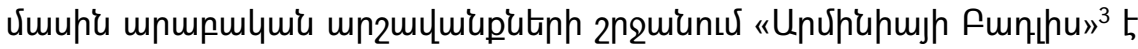

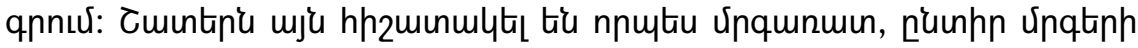

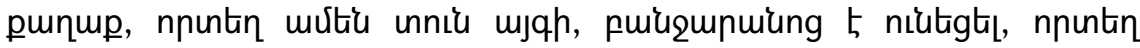
mutiu untumuly unnq 5 mati:

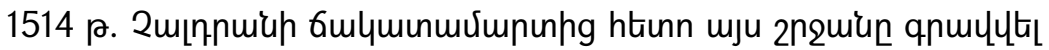

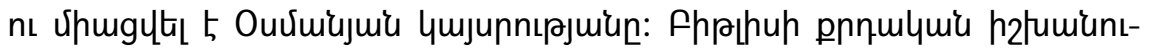

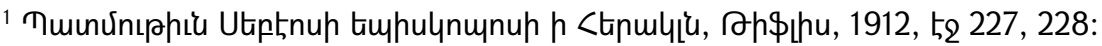

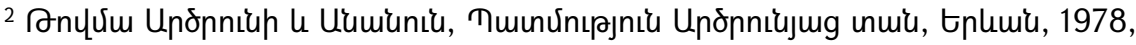
52 127:

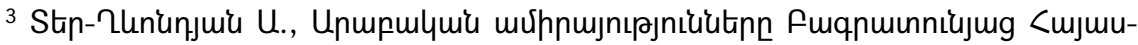
unuiunus, tplumiu, 1965, t52 230:
} 


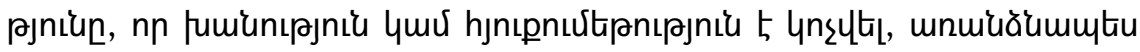

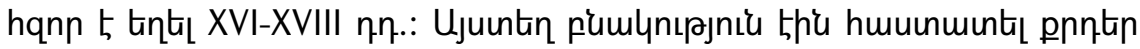
no tiqnhulitp:

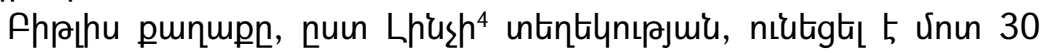

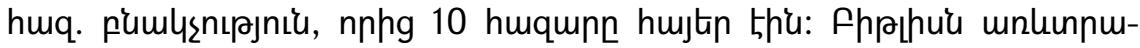

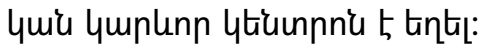

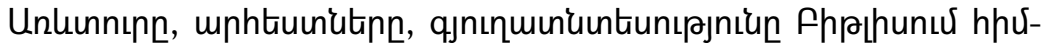

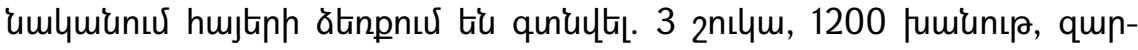

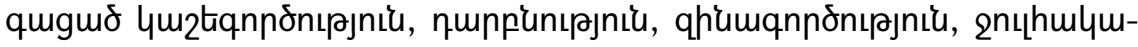

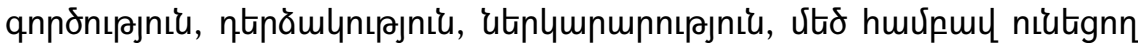

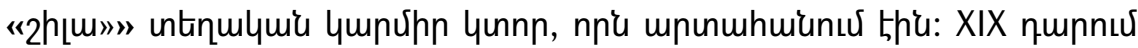

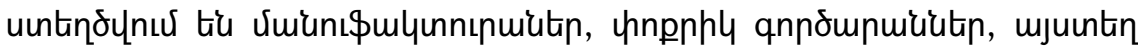

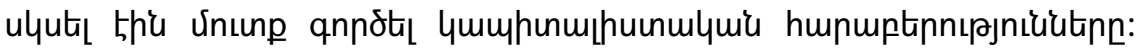

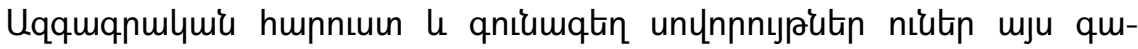
ywnn:

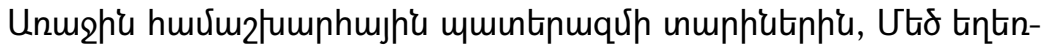

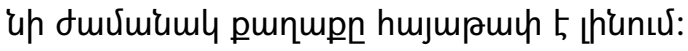

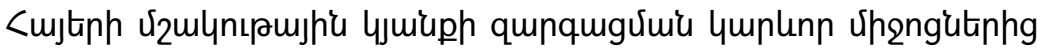

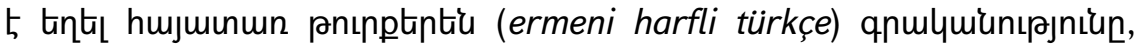

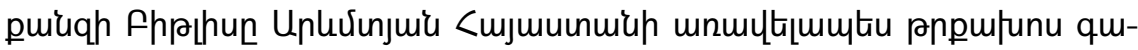

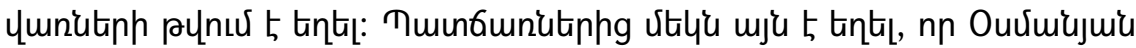

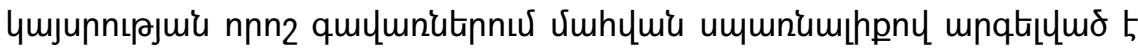

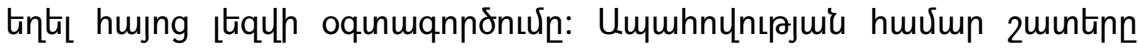

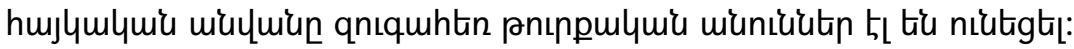

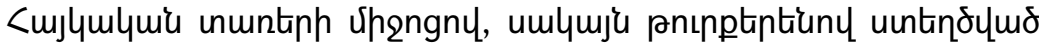

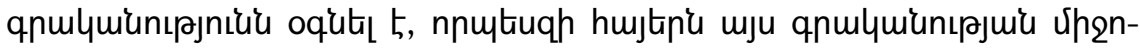

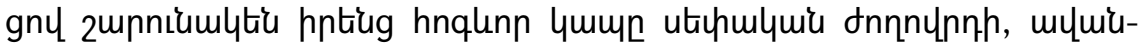

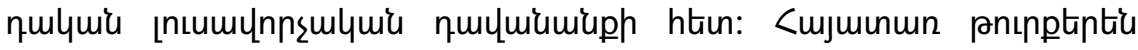

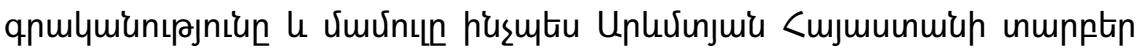

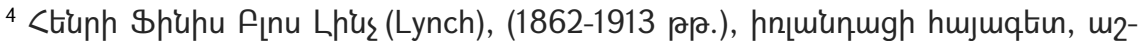

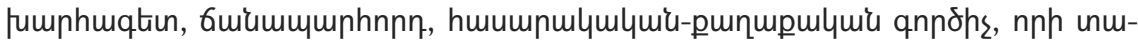
unn utpuuunugh hujnıhp 5 tint!: St' и Линч X. Ф. Б., Армения..., т. 2, Тифрлись, 1910, стр. 186-200.
} 


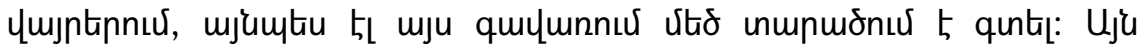

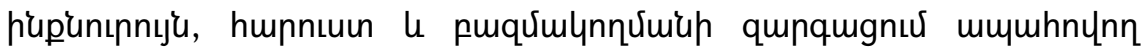

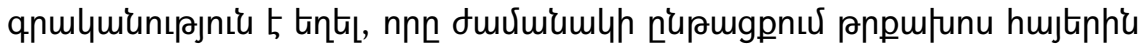

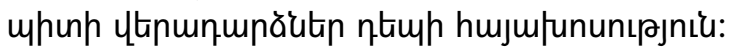

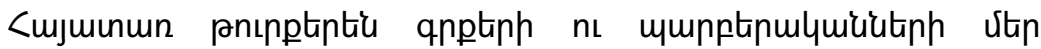

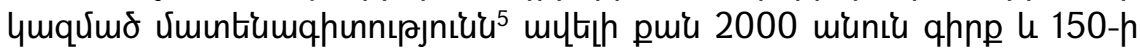

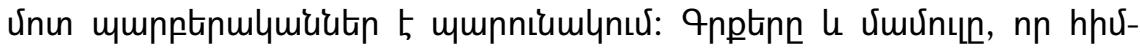

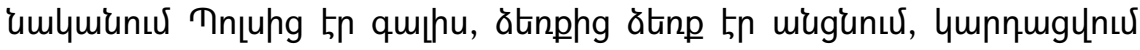

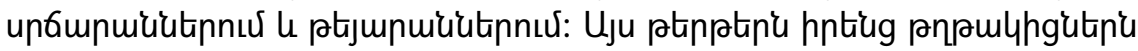

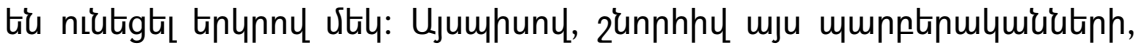

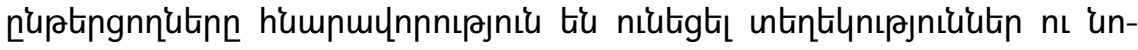

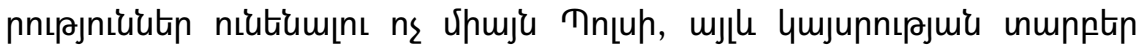

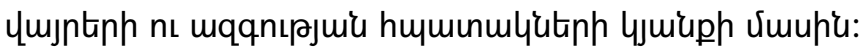

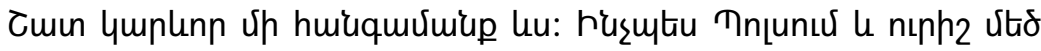

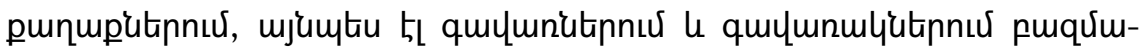

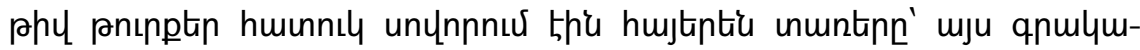

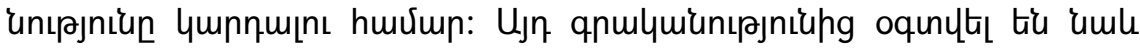

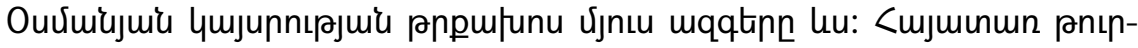

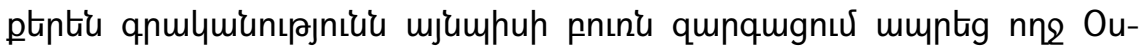

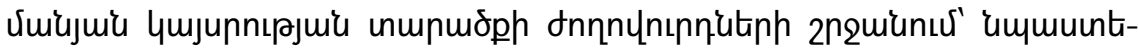

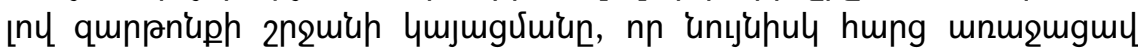

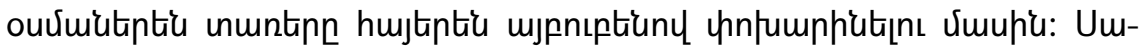

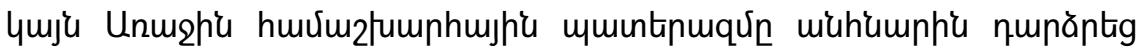

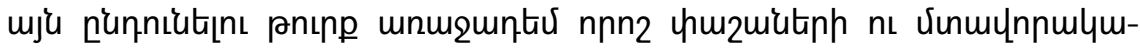
unıрJuiu quiuptinn:

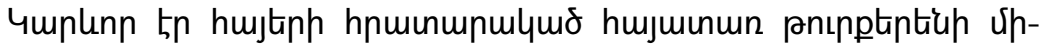

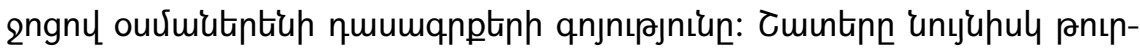

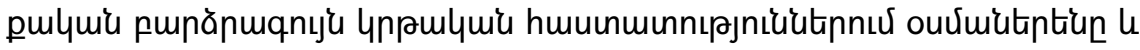

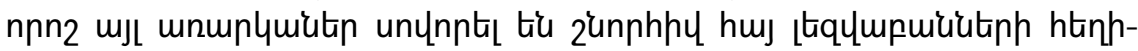
umlquठ hujuinum quumqpptiph:

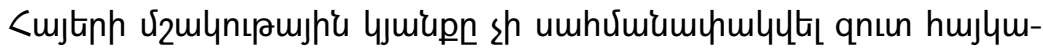

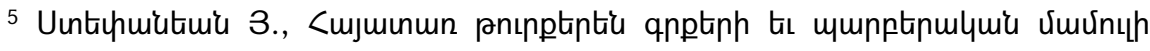
Uumutiumqhunnıphı̀u, Uınuúpnц, 2005: 


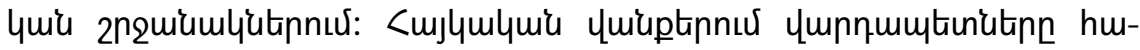

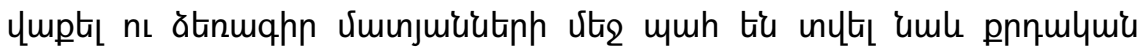

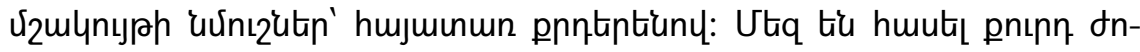

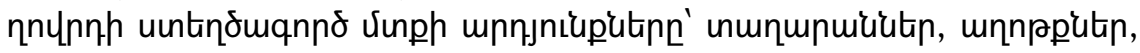

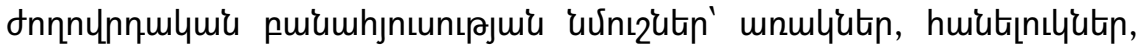

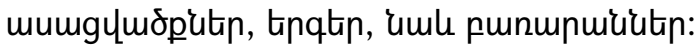

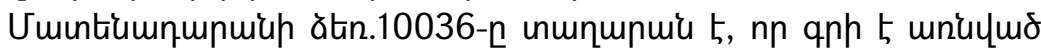

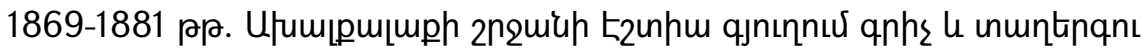

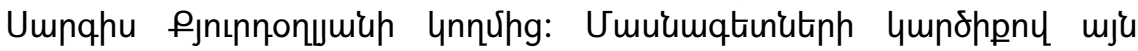

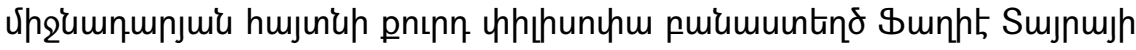
(Faqi Tayran (luuv Feqiyê Teyran (1590-1660 юр.) uuntnðuqnnont-

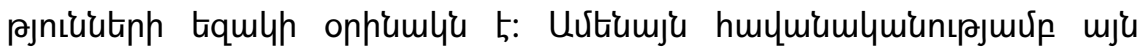

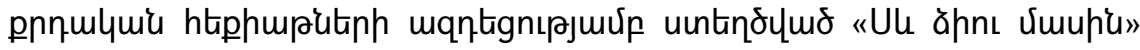

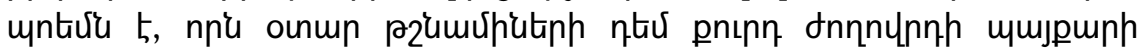

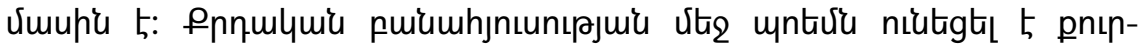

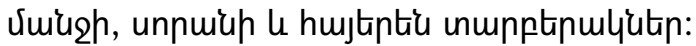

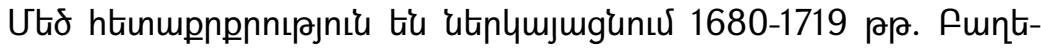

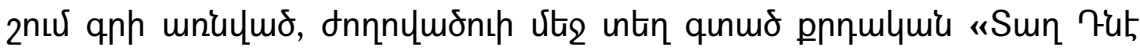

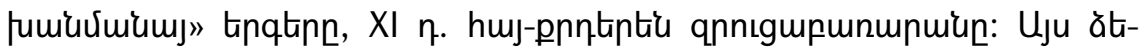
nuqhn dnnnumoniu qunulnuर 5 Фunhqh Uqqujhu qnunupuiuh huj-

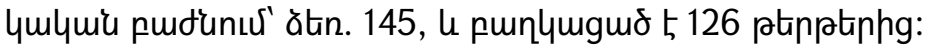

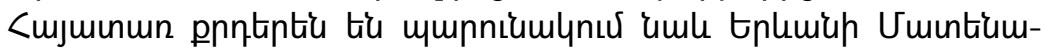
qupuiuh h. 737, 771, 4618, 5066, 7117, 8049, 10036 attnuqptang: Utiup

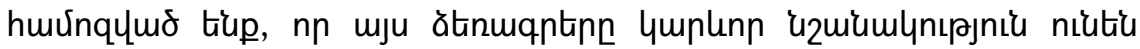

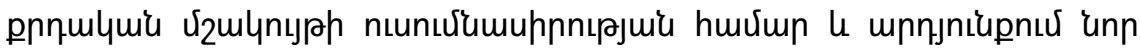

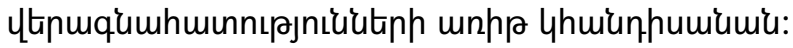

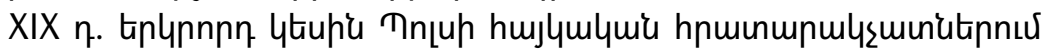

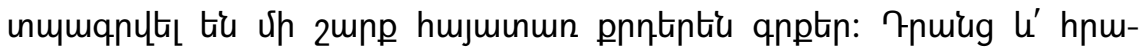

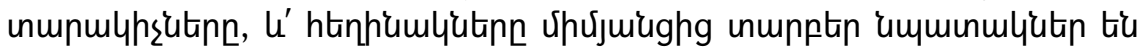
htinmumuntri:

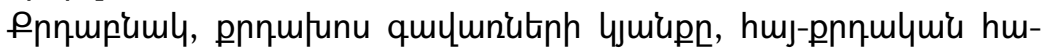

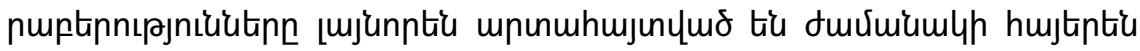

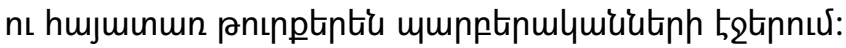

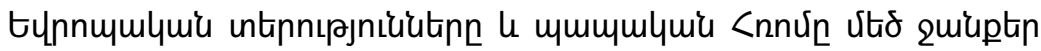




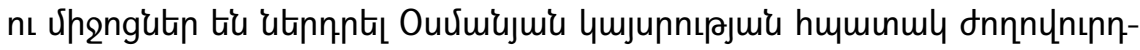

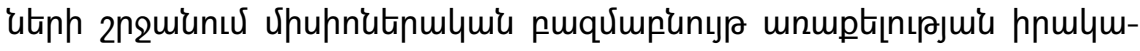

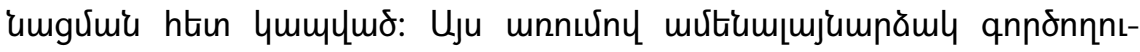

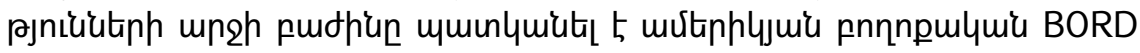

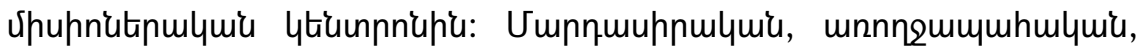

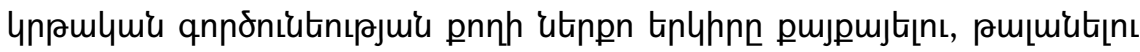
punupulquiunıs

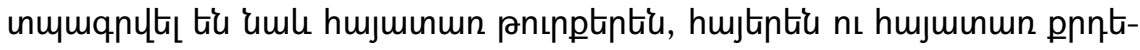

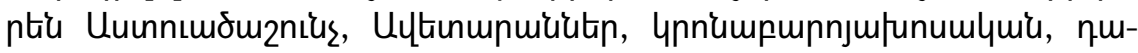
чuiampuiamlymí qnptip:

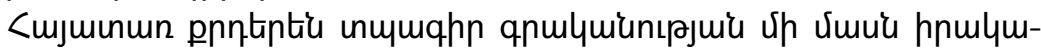

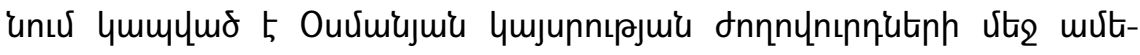

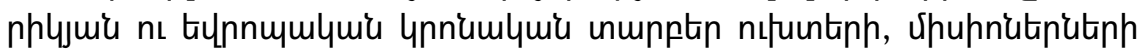

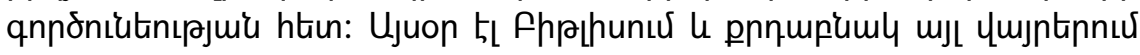

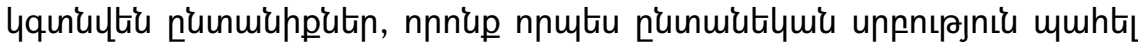

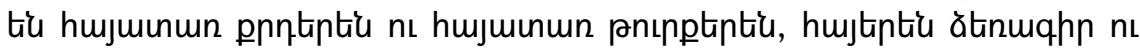

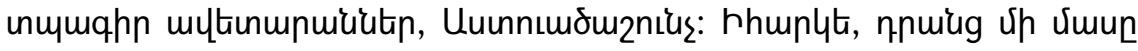

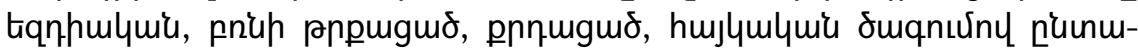
unputip cyting 5 hiutü:

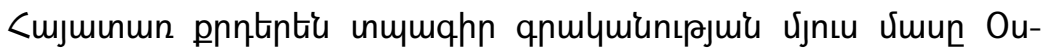

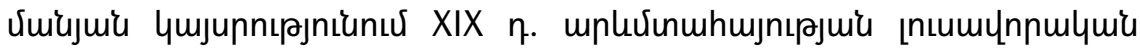

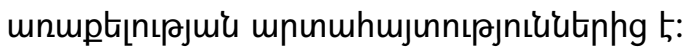

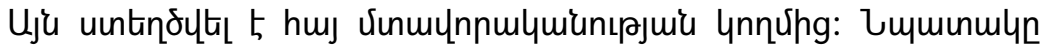

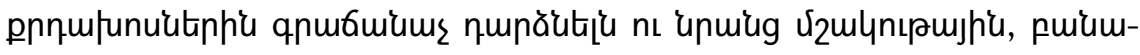

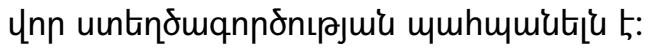

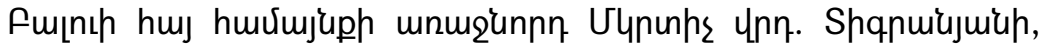

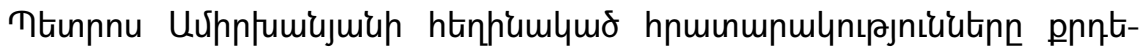

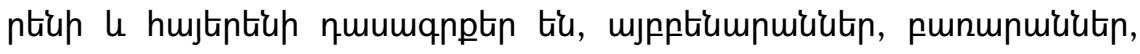

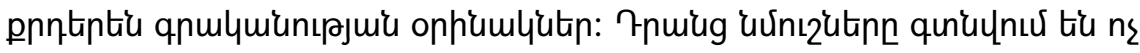

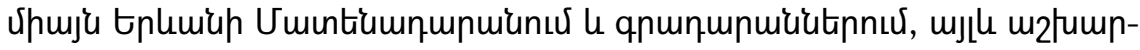

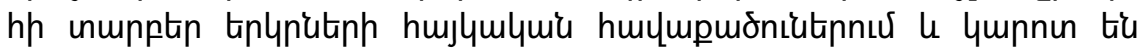

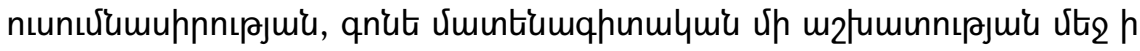
uh huчumpltint :

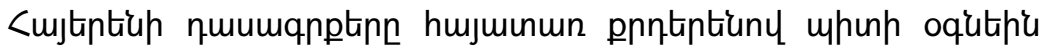




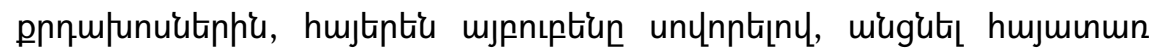
pnntintiup niunıgúuiu:

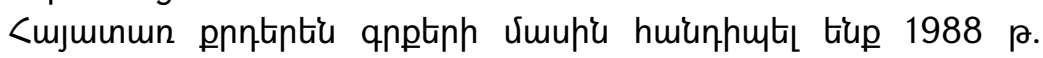

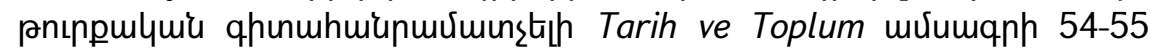

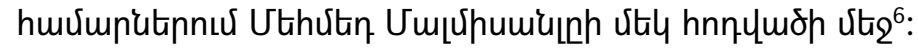

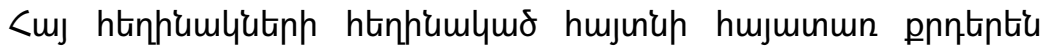

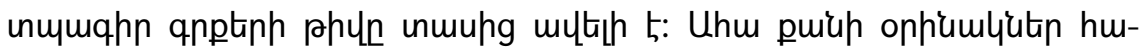

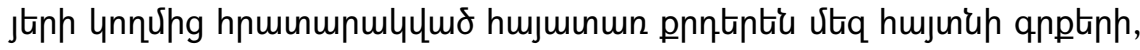

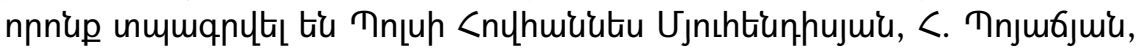

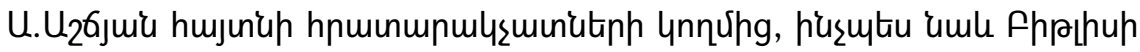
पhıujtipnuर:

1. Incil bi Qewlê Matteos. 4. Tnцhu, 1857:

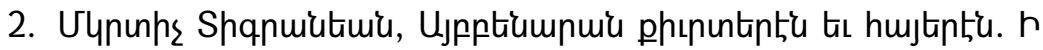

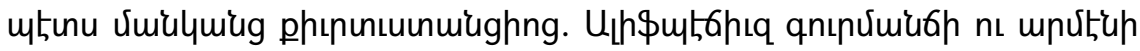
4. Tnцhu, unu. <. Ujnıhtiunhujuí, 1860,55 t:

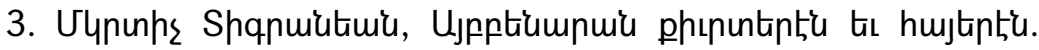

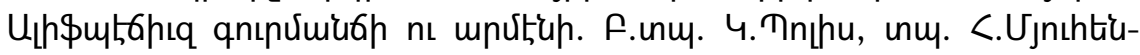
п.һujuiu, 1861, 55 t2: 1872:

4. Peynamê Noêxoiyê İsa-el-Mesihe. 4. Tnıhu, unщ. Tnjurjuưu,

5. İncila Rabbê Me Hisus Kristos ku Matteos Nivrisi. 4. Tnцhu, unu.

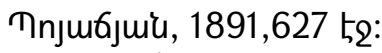

6. İncila Rabbê Me Hisus Kristos ku Margos Nivrisi. 4. Tnıhu, unu. Tnлшбјuiu, 1911, 87 5\&:

7. İncila Rabbê Me Hisus Kristos ku Xugas Nivrisi. 4. Tnıhu, unu.

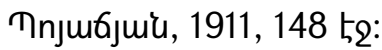

8. İncila Rabbê Me Hisus Kristos ku Hovhannes Nivrisi. 4. Tnцhu,

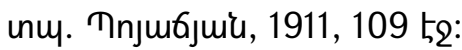

9. Emilê Resula. 4. Tnцhu, unu. Tnjuбjuiu, 1911,138 5,:

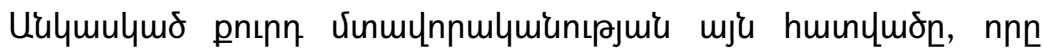

${ }^{6}$ Malmisanli Mehmet, Osmanli Döneminde Yazilan Ermeni Kürtçe Eserler Üzerine. Tarih ve Toplum, İst., 1988, N 54-55, s. 58-63. 


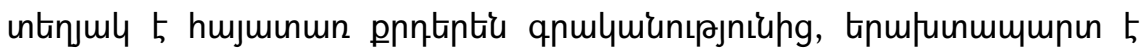

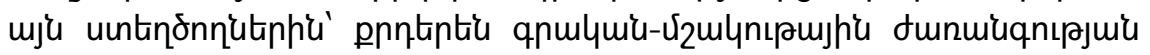

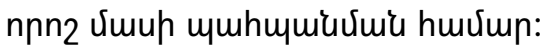

\section{STEPANYAN HASMIK}

\section{ARMENIANS IN BITLIS AND ARMENIAN KURDISH HANDWRITTEN AND PRINTED LITERATURE}

Bitlis is an ancient city where the Armenians were one of the oldest inhabitants. In 1878-79 the population of province was nearly 400.000, of which Armenians were 250.000. The population of Bitlis was 30.000, of which Armenians were 10.000. The Armenians had a great investment on the economic, trade and cultural life of the province as well as on the development of crafts and the penetration of the capitalistic relations.

Armenians lived mainly with Kurds and Yezidis. During the Genocide the Armenians were forced to leave Bitlis. One of the main cultural ways of Armenians was Armenian Turkish literature. There was also handwritten and printed literature in Armenian as well as in Kurdish. It was made by the Armenians for the Kurds keeping Kurdish folklore, philosophical and literary heritage. We could find published textbooks in Kurdish, the authors of which were Armenians.

Armenian Kurdish Bible, Gospels and other religious books were published by American missionaries. 